University of South Carolina

Scholar Commons

8-2003

\title{
Validation of a 3-Day Physical Activity Recall Instrument in Female Youth
}

Russell R. Pate

University of South Carolina - Columbia, rpate@mailbox.sc.edu

Rebecca Ross

Marsha Dowda

University of South Carolina - Columbia, mdowda@mailbox.sc.edu

Stewart G. Trost

John R. Sirard

Follow this and additional works at: https://scholarcommons.sc.edu/

sph_physical_activity_public_health_facpub

Part of the Public Health Commons

\section{Publication Info}

Published in Pediatric Exercise Science, Volume 15, Issue 3, 2003, pages 257-265.

Pate, R. R., Ross, R., Dowda, M., Trost, S. G., \& Sirard, J. R. (2003). Validation of a 3-day physical activity recall instrument in female youth. Pediatric Exercise Science, 15(3), 257-265.

(C) Pediatric Exercise Science, 2003, Human Kinetics

This Article is brought to you by the Physical Activity and Public Health at Scholar Commons. It has been accepted for inclusion in Faculty Publications by an authorized administrator of Scholar Commons. For more information, please contact digres@mailbox.sc.edu. 


\title{
Original Research
}

Pediatric Exercise Science, 2003, 15, 257-265

(C) 2003 Human Kinetics Publishers, Inc.

\section{Validation of a 3-Day Physical Activity Recall Instrument in Female Youth}

\author{
Russell R. Pate, Rebecca Ross, Marsha Dowda, \\ Stewart G. Trost, and John R. Sirard
}

\begin{abstract}
The purpose of this study was to examine the validity of the 3-Day Physical Activity Recall (3DPAR) self-report instrument in a sample of eighth and ninth grade girls ( $n=70,54.3 \%$ white, $37.1 \%$ African American). Criterion measures of physical activity were derived using the CSA 7164 accelerometer. Participants wore a CSA monitor for 7 consecutive days and completed the self-report physical activity recall for the last 3 of those days. Self-reported total METs, 30-min blocks of MVPA, and 30-min blocks of VPA were all significantly correlated with analogous CSA variables for 7 days $(r=0.35-$ $0.51 ; P<0.01)$ and 3 days $(r=0.27-0.46 ; P<0.05)$ of monitoring. The results indicate that the 3DPAR is a valid instrument for assessing overall, vigorous, and moderate to vigorous physical activity in adolescent girls.
\end{abstract}

\section{Introduction}

The development of accurate assessment tools for quantifying physical activity in children and adolescents continues to be a research priority $(13,22)$. Well-validated measures of youth physical activity are essential for determining the prevalence of physical activity in defined population groups, the efficacy of physical activity promotion programs, and the relationships between physical activity and health outcomes. To date, a wide range of methods has been used to measure physical activity in children and adolescents, including self-report questionnaires, direct observation, heart rate monitoring, accelerometry, and doubly labeled water. Of these, self-report questionnaires are typically used in population studies because they are characteristically non-reactive, practical (convenient and affordable), and can be customized to measure specific variables of interest $(9,15)$.

The 3-Day Physical Activity Recall (3DPAR) is a self-report instrument designed specifically for the recall abilities of adolescent youth. Using the same 30min time block format as the previously validated Previous Day Physical Activity

R.R. Pate, R. Ross, M. Dowda, and J.R. Sirard are with the Department of Exercise Science, School of Public Health, University of South Carolina, Columbia, SC; S.G. Trost is with the School of Human Movement Studies, University of Queensland, Brisbane, Australia. 
Recall (PDPAR; 20,24), the 3DPAR prompts respondents to recall the specific activities they engaged in, and the relative intensities of those activities. However, 3DPAR extends the PDPAR methodology by providing for the reporting of physical activity on 3 days during a single 30-min data collection session. Because 3DPAR assesses multiple days of physical activity, it may enable researchers to estimate "usual" physical activity. Also, because it is administered in a single reporting session, 3DPAR is particularly well suited for school-based investigations in which access to students is limited to one or two class periods.

The aim of the present study was to examine the validity of the 3DPAR selfreport instrument in a sample of eighth and ninth grade girls. Specifically, we assessed the validity of 3DPAR-derived estimates of total, moderate-to-vigorous, and vigorous physical activity using the Computer Science and Applications, Inc. (CSA) 7164 accelerometer to provide 7-day and 3-day criterion measures of physical activity.

\section{Methods}

\section{Subjects}

Subjects for this study were 70 eighth- and ninth-grade female students from two middle schools, two high schools, and a recreational soccer team in Columbia, South Carolina. Subject characteristics are reported in Table 1. Most of the participants were either white (54.3\%) or African American (37.1\%). All subjects were between the ages of 13 and 16 years (mean $\pm \mathrm{SD}, 14.0 \pm 0.9$ ). Prior to participation in the study, all subjects provided written assent to participate and their parents provided written informed consent. The study was approved by the University of South Carolina Institutional Review Board.

\section{Height and Weight}

Height was measured to the nearest $0.5 \mathrm{~cm}$ with a portable stadiometer, and weight was measured to the nearest $0.1 \mathrm{~kg}$ with a calibrated digital scale (BeFour, Inc.).

Table 1 Descriptive Data for Study Participants $(N=70)$

\begin{tabular}{ll}
\hline Characteristic & $\begin{array}{c}\text { Mean } \pm \text { SD } \\
\text { or Percent }\end{array}$ \\
\hline Age $(\mathrm{yr})$ & $14.0 \pm 0.9$ \\
Weight $(\mathrm{kg})$ & $58.6 \pm 15.0$ \\
Height $(\mathrm{m})$ & $1.61 \pm 0.06$ \\
BMI $\left(\mathrm{kg} / \mathrm{m}^{2}\right)$ & $22.4 \pm 5.4$ \\
\hline
\end{tabular}




\section{Self-Report Instrument}

The 3-Day Physical Activity Recall (3DPAR) instrument requires the recall of activity performed during each of the three previous days, beginning with the most recent day. Each day is segmented into thirty-four 30-min time blocks (7:00 am to midnight) which, in turn, are grouped into three broader time periods-morning, afternoon and evening. The instrument provides a list of 55 common activities grouped into the following categories: sleep/bathing, eating, work, after-school/ spare time/hobbies, transportation, and physical activities/sports. For each block of each day, participants entered the main activity in which they participated during each 30-min time period. Participants also rated the relative intensity of the designated activity as light, moderate, hard, or very hard. To help participants select the correct intensity level, the instrument provides pictorial representations of the four levels of relative intensity.

\section{Criterion Physical Activity Measure}

Objective assessments of physical activity were obtained using the Computer Science and Applications, Inc. (CSA) 7164 activity monitor (Shalimar, FL). The CSA 7164 is a uniaxial accelerometer designed to detect vertical acceleration ranging in magnitude from 0.05 to 2.0 Gs with a frequency response of 0.25 to $2.50 \mathrm{~Hz}$. These parameters allow for detection of normal human motion and will reject high frequency vibrations encountered in activities such as riding in a vehicle on a bumpy road. The filtered acceleration signal is digitized, and the magnitude is summed over a user-specified time interval. At the end of each interval, the summed value or "activity count" is stored in memory, and the integrator is reset. For the present study, a 1-min sampling interval was used. The CSA 7164 has been shown to be a valid and reliable tool for assessing physical activity in youth $(7,8,21)$.

\section{Protocol}

The study protocol was administered over a 7-day period. On the first day, groups of 20 or fewer participants were outfitted with a CSA 7164 activity monitor which was worn for the next 7 days. Consistent with previous investigations, monitors were worn over the right hip, anterior to the iliac crest. Participants were asked to wear the monitors at all times except during periods of sleeping, bathing, or swimming. Written instructions regarding the proper placement and handling of the monitors were provided to all participants. At the completion of the 7-day monitoring period (always a Wednesday), subjects completed the 3DPAR during an approximate 30-min period, recalling activity performed on the previous Tuesday, Monday, and Sunday. A trained research assistant administered the survey to groups of approximately 15 girls using a standardized procedure.

\section{Data Reduction}

3DPAR. Based on the specific activity and the level of intensity reported by the subject, each 30-min block was assigned a literature-based MET value (1). MET values were summed over each of the 3 days (Sunday, Monday, and Tuesday) for a measure of total daily physical activity (METs $\cdot$ day $^{-1}$ ). In addition, the 
number of 30-min blocks in which energy expenditure was estimated at 3 METs or greater (moderate-to-vigorous activity, MVPA) and 6 METs or greater (vigorous activity, VPA) were summed for each individual day. Three-day averages for METs · day $^{-1}$, MVPA blocks $\cdot$ day $^{-1}$, and VPA blocks $\cdot$ day $^{-1}$ were calculated.

In cases in which the reported activity type and intensity level were considered incompatible (e.g., the activity of sleeping and intensity rating of hard), the block in question was assigned a MET value considered appropriate for that activity. The standard 3DPAR protocol indicates that participants who make four or more incompatible responses should be excluded from the study. In this study, no participants were excluded because of incompatible responses.

CSA Activity Monitor. Stored minute-by-minute activity counts were uploaded to a customized software program for the determination of total daily activity counts and daily time spent in MVPA ( $\geq 3$ METs) and VPA ( $\geq 6$ METs). To match the recall period of the 3DPAR, only those counts recorded between 7:00 am and 12 midnight were examined. Each minute was categorized by intensity level (light, moderate, or vigorous) based on the number of counts recorded, using the regression equation developed by Freedson and colleagues (10). Single day totals, averages for the 3 days corresponding to the 3DPAR, and averages for the total 7 days of monitoring were calculated for counts, minutes of MVPA, and minutes of VPA.

\section{Statistical Analysis}

Pearson-product-moment correlation coefficients were calculated to evaluate associations between the CSA accelerometer variables and the corresponding 3DPAR variables. Averages of VPA minutes over 3 and 7 days of CSA monitoring were determined to be highly skewed and were log transformed for analyses. Statistical significance was set at an alpha level of 0.05 .

\section{Results}

Table 2 presents the means and standard deviations of the 3DPAR and CSA physical activity variables for individual days as well as the averages for 3 days. Also, for the CSA variables, the averages for 7 days of monitoring are presented. Over the 7 day period, the CSA monitors recorded less than 50 min of MVPA and less than 4 minutes of VPA daily. Some of the CSA and the 3DPAR variables suggest that the girls were less active on Sunday than on the two weekdays observed.

Table 3 shows the correlations between the 3DPAR and CSA variables, the latter expressed as averages for both 7 days and 3 days of monitoring. Self-reported total METs and CSA total counts were significantly correlated, with correlations of 0.51 and $0.46(P<0.001)$ for 7 and 3 days of monitoring, respectively. Self-reported blocks of MVPA and CSA minutes of MVPA showed correlations of $0.35(P<.01)$ for 7 days of monitoring and $0.27(P<.05)$ for 3 days of monitoring. The correlations between self-reported VPA and CSA minutes of VPA were 0.45 ( 7 days) and 0.41 ( 3 days $)(P<.001)$.

Pearson correlation coefficients for the associations between single day 3DPAR and CSA data are presented in Table 4. Correlations between self-reported total METs and CSA total counts ranged from $0.29(P<0.05)$ for Monday to 0.64 
Table 2 Means and Standard Deviations for 3DPAR and CSA Physical Activity Variables in 8th and 9th Grade Girls

\begin{tabular}{|c|c|c|}
\hline & 3DPA & RCSA \\
\hline & METs $\cdot$ day $^{-1}$ & Counts $\cdot$ day $^{-1}$ \\
\hline Tuesday & $64.4 \pm 14.4$ & $301,382 \pm 150,249$ \\
\hline Monday & $61.8 \pm 11.7$ & $296,783 \pm 141,450$ \\
\hline Sunday & $56.1 \pm 11.7$ & $229,472 \pm 125,050$ \\
\hline 3 day average & $60.8 \pm 9.1$ & $275,879 \pm 101,296$ \\
\hline \multirow[t]{2}{*}{7 day average } & & $296,921 \pm 96,135$ \\
\hline & $\begin{array}{c}\text { MVPA } \\
\left(\text { blocks } \cdot \text { day }^{-1}\right)\end{array}$ & $\begin{array}{c}\text { MVPA } \\
\left(\mathrm{min} \cdot \mathrm{day}^{-1}\right)\end{array}$ \\
\hline Tuesday & $3.7 \pm 3.1$ & $51.8 \pm 35.9$ \\
\hline Monday & $3.3 \pm 2.8$ & $51.0 \pm 33.4$ \\
\hline Sunday & $3.8 \pm 3.7$ & $32.9 \pm 30.9$ \\
\hline 3 day average & $3.6 \pm 2.3$ & $45.2 \pm 25.2$ \\
\hline \multirow[t]{2}{*}{7 day average } & & $49.4 \pm 23.3$ \\
\hline & $\begin{array}{c}\text { VPA } \\
\left(\text { blocks } \cdot \text { day }^{-1}\right)\end{array}$ & $\begin{array}{c}\text { VPA } \\
\left(\min \cdot \text { day }^{-1}\right)\end{array}$ \\
\hline Tuesday & $1.7 \pm 2.6$ & $4.0 \pm 8.0$ \\
\hline Monday & $1.2 \pm 1.8$ & $2.8 \pm 5.2$ \\
\hline Sunday & $0.8 \pm 1.6$ & $2.8 \pm 7.0$ \\
\hline 3 day average & $1.2 \pm 1.5$ & $3.2 \pm 5.6$ \\
\hline 7 day average & & $3.6 \pm 5.0$ \\
\hline
\end{tabular}

for Tuesday $(P<0.001)$. The correlations between self-reported MVPA and CSA minutes of MVPA were significant for Tuesday and Sunday; the values ranged from $0.32(P<0.05)$ for Sunday to $0.35(P<0.001)$ for Tuesday. The correlations between self-reported VPA and CSA minutes of VPA ranged from $0.30(P<0.05)$ for Sunday to $0.41(P<0.001)$ for Monday.

\section{Discussion}

This study evaluated the validity of the 3DPAR self-report instrument in an ethnically diverse sample of eighth and ninth grade girls. On average, girls participating in the study failed to meet current physical activity standards $(3,23)$. Our results indicate that the 3DPAR is a valid instrument for assessing vigorous, moderate to vigorous, and overall physical activity in this population. Similar to other 
Table 3 Pearson Correlation Coefficients for Associations Between

Means of 3DPAR and CSA Variables in 8th and 9th Grade Girls $(N=70)$

\begin{tabular}{|c|c|c|c|}
\hline \multirow[b]{2}{*}{ CSA Variables } & \multicolumn{3}{|c|}{ 3DPAR Variables } \\
\hline & $\begin{array}{l}\text { Total METs } \\
\left(\text { METS } \cdot \text { day }^{-1}\right)\end{array}$ & $\begin{array}{c}\text { MVPA } \\
\left(\text { Blocks } \cdot \text { day }^{-1}\right)\end{array}$ & $\begin{array}{c}\text { VPA } \\
\left(\text { Blocks } \cdot \text { day }^{-1}\right)\end{array}$ \\
\hline \multicolumn{4}{|l|}{7 days } \\
\hline Counts $\cdot$ day $^{-1}$ & $0.51 * * *$ & $0.38 * *$ & $0.55 * * *$ \\
\hline MVPA $\min \cdot$ day $^{-1}$ & $0.52 * * *$ & $0.35 * *$ & $0.56 * * *$ \\
\hline $\mathrm{VPA} \dagger \min \cdot \mathrm{day}^{-1}$ & $0.39 * * *$ & 0.19 & $0.45 * * *$ \\
\hline \multicolumn{4}{|l|}{3 days } \\
\hline Counts $\cdot$ day $^{-1}$ & $0.46^{* * * *}$ & $0.28 *$ & $0.46^{* * *}$ \\
\hline MVPA min $\cdot$ day $^{-1}$ & $0.47 * * *$ & $0.27 *$ & $0.47 * * *$ \\
\hline $\mathrm{VPA} \dagger \min \cdot \mathrm{day}^{-1}$ & $0.36 * *$ & 0.17 & $0.41 * * *$ \\
\hline
\end{tabular}

$* P<0.05 ; * * P<0.01 ; * * * P<0.001 ; \dagger$ Log transformed.

Table 4 Pearson Correlation Coefficients Between the 3DPAR and CSA Variables for Individual Days $(N=70)$

\begin{tabular}{|c|c|c|c|}
\hline \multirow[b]{2}{*}{ CSA Variables } & \multicolumn{3}{|c|}{ 3DPAR Variables } \\
\hline & $\begin{array}{c}\text { Total METs } \\
\left(\text { METS } \cdot \text { day }^{-1}\right)\end{array}$ & $\begin{array}{c}\text { MVPA } \\
\left(\text { Blocks } \cdot \text { day }^{-1}\right)\end{array}$ & $\begin{array}{c}\text { VPA } \\
\left(\text { Blocks } \cdot \text { day }^{-1}\right)\end{array}$ \\
\hline \multicolumn{4}{|l|}{ Counts $\cdot$ day $^{-1}$} \\
\hline Tuesday & $0.64 * * *$ & $0.45 * * *$ & $0.67 * * *$ \\
\hline Monday & $0.29 *$ & 0.24 & 0.23 \\
\hline Sunday & $0.39 * *$ & $0.30 *$ & $0.34 * *$ \\
\hline \multicolumn{4}{|c|}{ MVPA min $\cdot$ day $^{-1}$} \\
\hline Tuesday & $0.59 * * *$ & $0.35 * * *$ & $0.59 * * *$ \\
\hline Monday & $0.33 * *$ & 0.23 & $0.28^{*}$ \\
\hline Sunday & $0.43 * * *$ & $0.32 *$ & $0.42 * * *$ \\
\hline \multicolumn{4}{|c|}{$\mathrm{VPA} \dagger \min \cdot \mathrm{day}^{-1}$} \\
\hline Tuesday & $0.37 * *$ & 0.14 & $0.41 * *$ \\
\hline Monday & $0.27 *$ & 0.11 & $0.32 *$ \\
\hline Sunday & 0.25 & 0.18 & $0.30 *$ \\
\hline
\end{tabular}

$* P<0.05 ; * * P<0.01 ; * * * P<0.001 ; \dagger$ Log transformed. 
self-report instruments, the 3DPAR demonstrated higher validity for assessment of vigorous physical activity than for expressions of physical activity that included lower intensities of activity. This probably reflected a better ability to recall participation in the more vigorous forms of activity (e.g., basketball) than those that are more moderate in intensity (e.g., walking). Self-reported MVPA and CSA minutes of MVPA were more highly correlated on Tuesday and Sunday, possibly because the self-report instrument was always administered on a Wednesday, which facilitated recall of Tuesday activities, and because Sunday is a weekend day and may be more distinct and easier to recall.

The validity coefficients observed for the 3DPAR compare favorably with those previously reported by our group for the original PDPAR instrument. We observed higher coefficients than reported by Trost and colleagues for single-day administration of PDPAR in fifth grade students (20). In that study, the correlation between self-reported average MET level and CSA accelerometer counts was 0.39, while self-reported blocks of vigorous activity exhibited a correlation of 0.35 with CSA counts. The higher values observed in the present study may be explained by the older age of the subjects. In contrast, the validity coefficients in this study were somewhat lower than those reported by Weston et al. (24). In that study, the correlation between total accelerometer counts and PDPAR-derived estimates of afterschool energy expenditure was 0.77 , while self-reported blocks of MVPA exhibited a correlation of 0.63 with the number of minutes at a heart rate greater than $50 \%$ of heart rate reserve. The higher values observed by Weston et al. are probably explained by the substantially shorter recall period (single afternoon vs. three complete days) and the inclusion of older high school students in the study sample.

The 3DPAR yielded validity coefficients that were somewhat higher than those of previous studies using accelerometers to validate self-report instruments in children and adolescents. Validity coefficients from studies using one-day recalls of physical activity compared to one day of activity monitoring range from 0.19 to $0.88(16,18,20,24)$. The one-day (Tuesday) correlations observed in this study ( 0.35 to 0.64$)$ are comparable to those previously reported. Janz et al. (12) reported correlations of 0.05 to 0.39 for a 3 -day sweat recall and 0.46 to 0.51 for a 3-day aerobic recall. The 3-day correlations presented in Table 3, ranging from 0.27 to 0.46 for analogous variables, are greater than those reported for the sweat recall and similar to the aerobic activity recall. Lastly, correlations between 7-day recalls or measures of self-reported "usual" physical activity and activity monitoring range from -0.26 to $0.47(6,11,17,14)$. Again, correlations from the present study ( 0.35 to 0.51$)$ are comparable to or slightly greater than those previously reported.

A unique characteristic of the 3DPAR is that it provides information about three days of physical activity through a report that is made in a single 30 -min session. Although no previous studies have determined the number of days of selfreport that are required to characterize usual physical activity, Trost and colleagues have shown that multiple days of activity monitoring are required to reliably characterize usual physical activity participation in children and youth (19). The data in this study show that 3DPAR variables generally correlated more highly with accelerometer data recorded over the preceding seven days than those recorded only during the three days corresponding directly to the 3DPAR report. This observation suggests that 3DPAR provides a valid reflection of both usual physical 
activity, which is better represented by seven days of monitoring than by three days, and physical activity performed during the specific recall period.

The present study documents the validity of the 3DPAR in adolescent girls. The study was undertaken prior to an investigation of physical activity promotion in this demographic group, because a self-report measure of physical activity was needed for use in that investigation. It is particularly important that valid measures of physical activity be available for use in studies of this age and gender group, since previous studies have shown that physical activity levels are lower in girls than boys and physical activity tends to decline rapidly in girls during adolescence $(2,4,5)$. Therefore, a valid self-report instrument such as the 3DPAR, which provides information on overall physical activity as well as participation in specific forms of physical activity and sedentary behaviors, enables research on an important public health problem. Nonetheless, the findings of this study cannot be generalized to boys or to youth in other age groups, and we recommend that future studies examine the validity of 3DPAR in diverse groups of youth.

This study was limited by the availability of only a single type of criterion measure, accelerometry. While accelerometers have been shown to provide useful estimates of physical activity in youth, physical activity is a complex behavior and no single measure constitutes a perfect "gold standard." For example, it is known that accelerometry does not accurately assess energy expenditure during non-weightbearing activities such as swimming and cycling and is insensitive to non-locomotor activities such as carrying loads. Accordingly, in future studies it would be desirable to validate 3DPAR against multiple criterion measures such as heart rate in addition to motion detected by accelerometry.

In conclusion, the 3DPAR was found to be a valid measure of physical activity in eighth and ninth grade girls. Results of this study specifically demonstrated that 3DPAR provides a valid reflection of usual physical activity as measured by accelerometry over a seven-day period, as well as during the three-day period corresponding to the recall.

\section{References}

1. Ainsworth, B.E., W.L. Haskell, M.C. Whitt, et al. Compendium of Physical Activities: An update of activity codes and MET intensities. Med. Sci. Sports Exerc. 32:S498S516, 2000.

2. Andersen, R.E., C.J. Crespo, S.J. Bartlett, L.J. Cheskin, and M. Pratt. Relationship of physical activity and television watching with body weight and level of fatness among children: Results from the third National Health and Nutrition Examination Survey. JAMA 279:938-942, 1998.

3. Biddle, S., J.F. Sallis, and N.A. Cavill. Young and active? Young people and health enhancing physical activity. Evidence and implication. London: Health Education Authority, 1998.

4. Centers for Disease Control and Prevention. Youth Risk Behavior Surveillance - United States, 1997. MMWR Morb. Mortal Wkly. Rep. 47:1-89, 1998.

5. Centers for Disease Control and Prevention. Youth Risk Behavior Surveillance - United States, 1999. MMWR Morb. Mortal Wkly. Rep. 49:1-95, 2000.

6. Craig, S.B., L.G. Bandini, A.H. Lichtenstein, E.J. Schaefer, and W.H. Dietz. The impact of physical activity on lipids, lipoproteins, and blood pressure in preadolescent girls. Pediatrics 98:389-395, 1996. 
7. Eston, R.G., A.V. Rowlands, and D.K. Ingledew. Validity of heart rate, pedometry, and accelerometry for predicting the energy cost of children's activities. J. Appl. Physiol. 84:362-371, 1998.

8. Fairweather, S.C., J.J. Reilly, S. Grant, A. Whittaker, and J.Y. Paton. Using the Computer Science and Applications (CSA) activity monitor in preschool children. Pediatr. Exerc. Sci. 11:413-420, 1999

9. Freedson, P.S., and E.L. Melanson. Measuring physical activity. In: Measurement in Pediatric Exercise Science, D. Docherty (Ed.). Champaign, IL: Human Kinetics, 1996, pp. 161-182.

10. Freedson, P.S., J. Sirard, E. Debold, R.R. Pate, M. Dowda, S.G. Trost, and J.F. Sallis. Calibration of the Computer Science and Applications, Inc. (CSA) accelerometer. Med. Sci. Sports Exerc. 29:S45, 1997.

11. Goran, M.I., G. Hunter, T.R. Nagy, and R. Johnson. Physical activity related energy expenditure and fat mass in young children. Int. J. Obes. 21:171-178, 1997.

12. Janz, K.F., J. Witt, and L.T. Mahoney. The stability of children's physical activity as measured by accelerometry and self-report. Med. Sci. Sports Exerc. 27:1326-1332, 1995.

13. Kohl, H.W., and K.E. Hobbs. Development of physical activity behaviors among children and adolescents. Pediatrics 101:549-554, 1998.

14. Kowalski, K.C., P.R.E. Crocker, and R.A. Faulkner. Validation of the physical activity questionnaire for older children. Pediatr. Exerc. Sci. 9:174-186, 1997.

15. Kriska, A.M. Introduction to a collection of physical activity questionnaires. Med. Sci. Sports Exerc. 29:S5-S9, 1997.

16. McMurray, R.G., J.S. Harrell, C.B. Bradley, J.P. Webb, and E.M. Goodman. Comparison of a computerized physical activity recall with a triaxial motion sensor in middleschool youth. Med. Sci. Sports Exerc. 30:1238-1245, 1998.

17. Sallis, J.F., M.J. Buono, J. Roby, F.G. Micale, and J.A. Nelson. Seven-day recall and other physical activity self-reports in children and adolescents. Med. Sci. Sports Exerc. 25:99-108, 1993.

18. Sallis, J.F., P.K. Strikmiller, D.W. Harsha, H.A. Feldman, S. Ehlinger, E.J. Stone, J. Williston, and S. Woods. Validation of interviewer- and self-administered physical activity checklists for fifth grade students. Med. Sci. Sports Exerc. 28:840-851, 1996.

19. Trost, S.G., R.R. Pate, P.S. Freedson, J.F. Sallis, and W.C. Taylor. Using objective physical activity measures with youth: How many days of monitoring are needed? Med. Sci. Sports Exerc. 32:426-431, 2000.

20. Trost, S.G., D.S. Ward, B. McGraw, and R.R. Pate. Validity of the Previous Day Physical Activity Recall (PDPAR) in fifth-grade children. Pediatr. Exerc. Sci. 11:341-348, 1999.

21. Trost, S.G., D.S. Ward, S.M. Moorehead, P.D. Watson, W. Riner, and J.R. Burke. Validity of the Computer Science and Applications (CSA) activity monitor in children. Med. Sci. Sports Exerc. 30:629-633, 1998.

22. U.S. Department of Health and Human Services. Physical Activity and Health: A Report of the Surgeon General. Atlanta: USDHHS/CDC, 1996.

23. U.S. Department of Health and Human Services. Healthy People 2010 (2nd ed.). Washington, DC: USDHHS, 2000.

24. Weston, A.T., R. Petosa, and R.R. Pate. Validity of an instrument for measurement of physical activity in youth. Med. Sci. Sports Exerc. 29:138-143, 1997. 